\title{
VAC.04 - Evaluation of humoral response of individuals naturally exposed to malaria against synthetic and recombinant antigens representing Plasmodium vivax TRAP protein
}

Ada da Silva Matos ${ }^{1 *}$; Rodrigo Nunes Rodrigues da Silva ${ }^{1}$; Isabela Ferreira Soares ${ }^{1}$; Barbara de Oliveira Baptista ${ }^{1}$; Paulo Renato Rivas Totino ${ }^{1}$; Claudio Tadeu Daniel-Ribeiro ${ }^{1}$; Cesar Lopez Camacho $^{2}$; Arturo Reyes-Sandoval ${ }^{2}$; Lilian Rose Pratt-Riccio ${ }^{1}$; Josué da Costa Lima Junior ${ }^{1}$.

1Fiocruz - Fundação Oswaldo Cruz;

2University of Oxford, United Kingdom.

Introduction: Thrombospondin-related adhesive protein (TRAP) of malaria parasites is essential for sporozoite motility and invasions into mosquito's salivary gland and vertebrate's hepatocyte. Due this importance, it is a promising target for pre-erythrocytic vaccine. Despite few reports on naturally acquired immune response against Plasmodium vivax TRAP (PvTRAP) are available, the results are conflicting and poorly explored in the Amazon region.

Objective: Characterize the humoral response to PvTRAP in exposed populations and explore it with indicators of exposure and protection.

Methodology: Therefore, we aimed to characterize the antibody reactivity (IgG and IgG subclass) against recombinant PvTRAP in a cross-sectional study of 299 individuals naturally exposed to malaria infections living in 3 communities in Brazilian Amazon (Cruzeiro do Sul, Mancio Lima and Guajara). Moreover the complete PvTRAP sequence were also screened for linear B-cell epitopes using in silico and in vitro approaches.

Results: Firstly, we confirmed that PvTRAP is naturally immunogenic in studied individuals, since $49 \%$ of studied population were IgG-responders against PvTRAP. The observed immune response was mainly drived by cytophilic antibodies IgG1 over all other sublcass. In addition, the IgG1 levels presented correlation with age and time of residence in endemic area $(\mathrm{p}<0.05)$. Interestingly, only the levels of specific anti-TRAP IgG3 seem to be associated with protection. Regarding the epitope mapping, four epitopes were predicted and confirmed as immunogenic among PvTRAP IgG-responders: R197-H227 (26\%), E237-T258 (25\%), P344-G374 (32\%) and E439-460 (29\%). However, none of the confirmed B-cell epitopes were associated with exposure or protection parameters.

Conclusion: PvTRAP is naturally immunogenic in populations exposed to malaria and exposure-associated IgG antibodies confirm its potential as vaccine candidate. The protein presents at least 4 linear B-cell epitopes in the full-length sequence, but the low frequency of responders and the lack of association between IgG levels against peptides and exposure and/ or protection, suggest that the main epitopes are conformational. Therefore, our data suggest that a recombinant vaccine based on PvTRTAP should be more effective than a peptide-based vaccine.

Keywords: Malaria; Humoral immune response; PvTRAP 